\title{
AGING AND MASTICATORY INFLUENCES ON SPATIAL LEARNING AND MEMORY AND ON ASTROCYTES MORPHOLOGY OF MOUSE DENTATE GYRUS MOLECULAR LAYER
}

F.B.A. Albuquerque1; F.C.C. Siqueira Mendes ${ }^{1,2}$; L.T.V.B. Paixao1; C.W.P. Diniz; M.C.K. Sosthenes ${ }^{1}$

${ }^{1}$ Federal University of Para, Laboratório de Investigações em Neurodegeneração e Infecção - ICB- HUJBB, Belém, Brazil.

2 Centro Universitário do Estado do Pará, Curso de Medicina, Belém, Brazil.

\section{Introduction and Methods}

Evidences indicate a reduction in masticatory activity as detrimental to memory. Thus to measure possible influences of masticatory changes on spatial learning and on the morphology of the dentate gyrus astrocytes in an aged murine model, we imposed one of three diet regimens on different experimental groups, from the $21^{\text {st }}$ postnatal day onwards until $6(6 \mathrm{M})$ or 18 months (18M) of life. To that end the control group with normal masticatory activity received a pellet-type hard diet; the group with reduced masticatory activity received a pellet diet followed by a powdered diet; and the group with rehabilitated masticatory activity received pelletized diet, followed by powder and pellet again. The changing intervals were proportional and the same for each diet. At $6 \mathrm{M}$ or $18 \mathrm{M}$ they were sacrificed for perfusion with saline and aldehyde fixatives (Figure 1).

\section{Results}

To measure the effects of diet and age on learning and spatial memory, we analyzed the individuals performance on the Morris water maze task and found that masticatory activity reduction decreased the average rate of spatial learning, and its rehabilitation recovered associated losses in young animals. No differences were found among old animals. No correlation was found between the learning rate and swimming speed, suggesting that deficits and their recovery reflect cognitive performance.

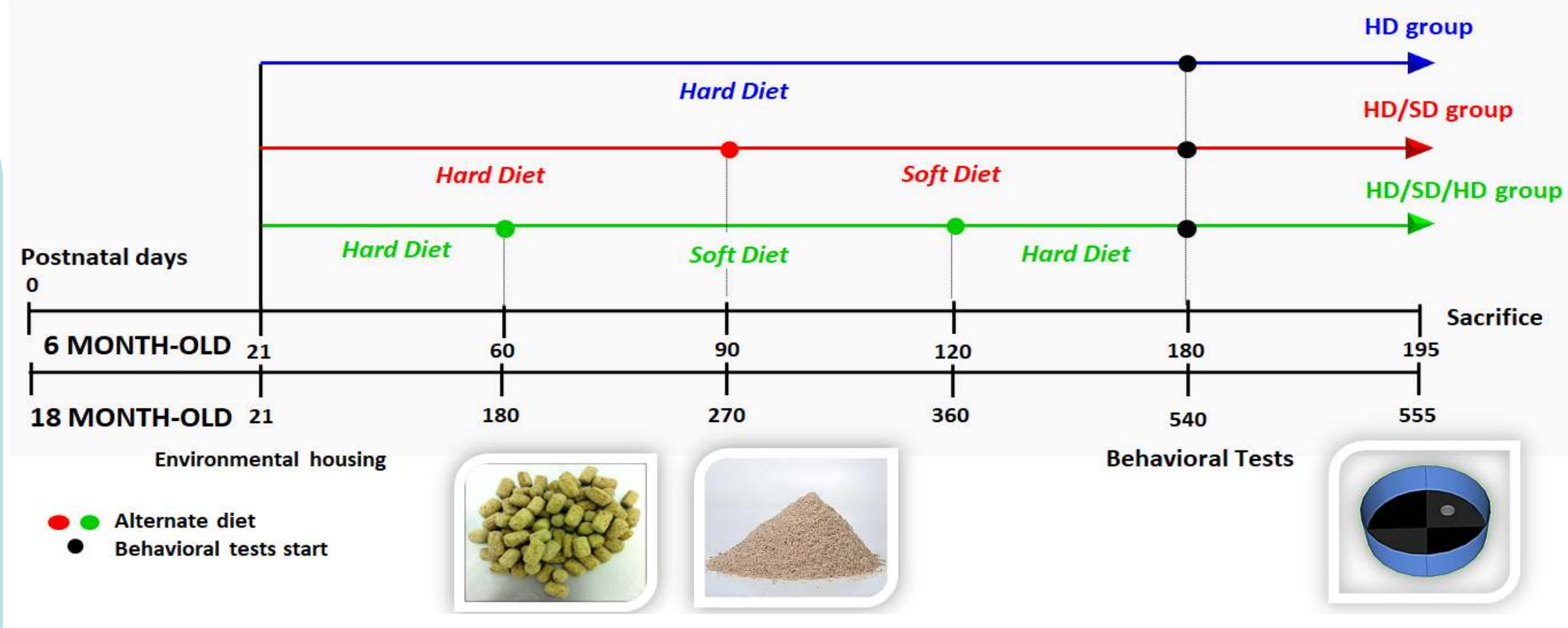

Figure 1: Sequence of events from the experimental model, highlighting the difference between food (image below) that alternated at different times according to the age of the animals in some experimental groups (HD/SD and HD/SD/HD).

For the morphological studies we used selective astrocyte immunolabeling directed to glial acid fibrillar protein (GFAP), reconstructing microscopically in three dimensions, those located in the outer one-third of the molecular layer of the dentate gyrus. 900 cells were digitally reconstructed and the morphological study using hierarchical cluster analysis revealed two morphological phenotypes, designated as type I and II that were differentially affected by the studied variables. Aging reduced the complexity morphology of astrocytic trees in type I, but no in type II, while masticatory change reduced only in young animals, increasing the complexity of the branches in old animals both type I and II (Figure 2). We conclude that reduction of masticatory activity and aging in mice impairs spatial learning in Morris water maze and the rehabilitation of masticatory activity seems to recover these losses in young mice. Although not linearly, the morphological changes suggest influence of chewing alteration on the astrocytes of the dentate gyrus.

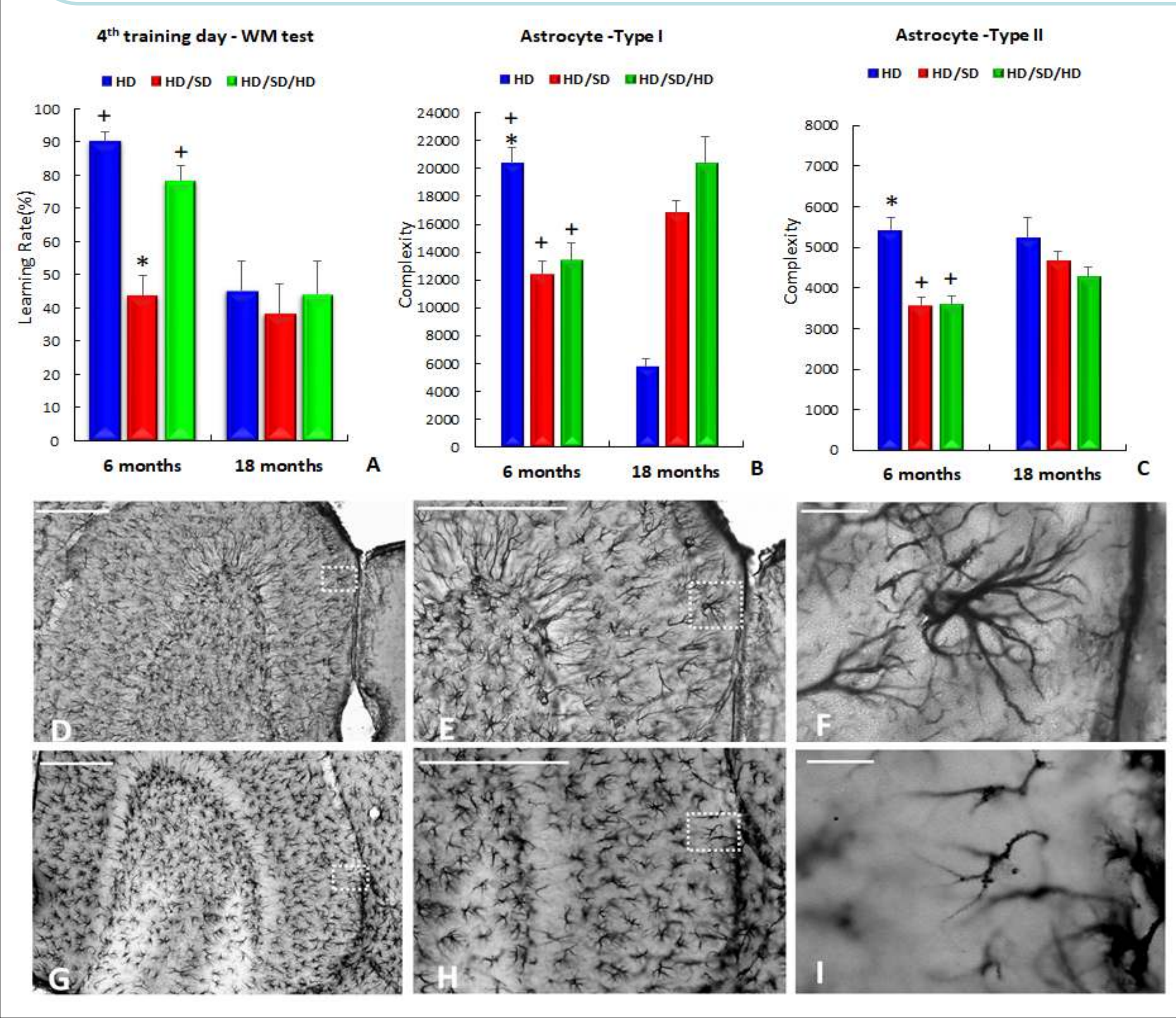

Figure 2: A: Water maze learning rate for all experimental groups. $\mathrm{B}$ and $\mathrm{C}$ : measure of the complexity of the branches of Type I (high complexity) and Type II (low complexity) astrocytes, respectively in all experimental groups. D, E and F: Photomicrograph of the brain section of the Mus musculus species immunostained with anti-GFAP antibody. Astrocytes located on the outer $1 / 3$ of the molecular layer of the dentate gyrus are visualized. The dotted square in image $D$ highlights a Type I astrocyte. $\mathrm{E}$ and $\mathrm{F}$ highlight the same cell through larger magnifying lenses. $\mathrm{G}, \mathrm{H}$ and $\mathrm{I}$ : follow the previous logic, revealing a type II astrocyte. Scale bars: $A=250 \mu \mathrm{m} ; B=250 \mu \mathrm{m} ; C=25 \mu \mathrm{m}$.
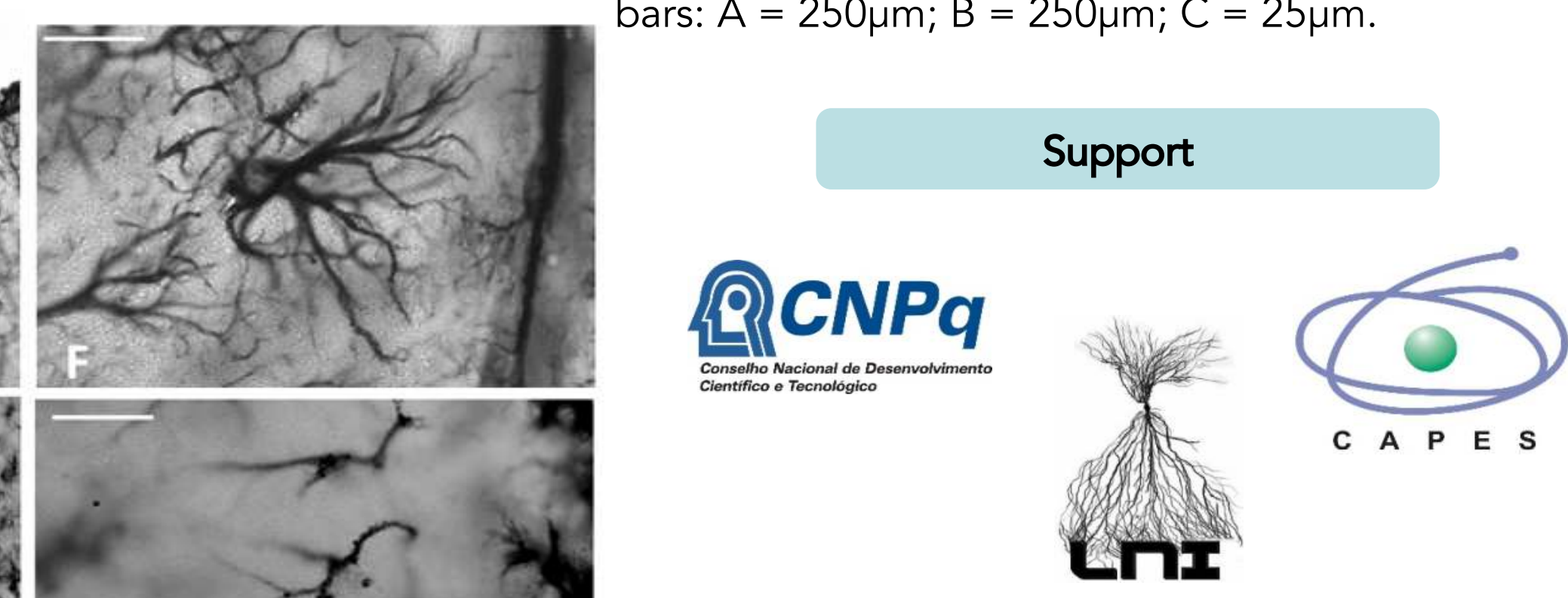